[1]

${ }^{1}$ Respiratory and GIM, Dept of Respiratory Medicine, Great Western Hospital NHS Foundation Trust, Swindon, UK. ${ }^{2}$ Respiratory and GIM, Musgrove Park Hospital, Taunton and Somerset NHS Foundation Trust, Taunton, UK. ${ }^{3} \mathrm{CU}$ and Anaesthetics, Musgrove Park Hospital, Taunton and Somerset NHS Foundation Trust, Taunton, UK.

Cite as: Hameed F, Pepperell J, Sidney J. A rare complication of endoscopic intervention. Breathe 2018; 14: e40-e42.

\title{
A rare complication of endoscopic intervention
}

\section{Case report}

A 56-year-old woman with alcohol-related liver cirrhosis presented with haematemesis and melena. On arrival, observations were oxygen saturation measured by pulse oximetry $97 \%$ on room air, blood pressure $77 / 44 \mathrm{mmHg}$, heart rate 127 per min, respiratory rate 24 per $\mathrm{min}$, temperature $36.5^{\circ} \mathrm{C}$ and alert. The blood tests showed haemoglobin $65 \mathrm{~g} \cdot \mathrm{L}^{-1}$ and urea $23 \mathrm{mg} \cdot \mathrm{dL}^{-1}$. After initial resuscitation with intravenous fluids, she was given a transfusion of 2 units packed red cells, 2 units fresh frozen plasma, 1 unit platelets and $2 \mathrm{mg}$ terlipressin.

\section{Task 1}

What is the next best management option?

a) Emergency oesophagogastroduodenoscopy

b) Emergency laparotomy

c) No further action required

\section{Answer 1}

a) Emergency oesophagogastroduodenoscopy

This patient presented with acute upper gastrointestinal bleeding and circulatory shock. After initial resuscitation, the next best management option would be upper gastrointestinal endoscopy for diagnostic and therapeutic purposes.

The emergency upper gastrointestinal endoscopy was carried out in the operating theatre under ventilation support via an endotracheal tube. Gastric and oesophageal varices were seen and injected with histoacryl/lipiodol (1/3) glue to effectively stop the bleeding. Within minutes of the injection of glue into the gastric varix, the patient dropped oxygen saturation and went into pulseless electrical activity (PEA) cardiac arrest.

\section{Task 2}

What are the possible causes of PEA cardiac arrest in this patient?
a) Hypovolaemia
b) Pulmonary embolic event
c) Electrolyte imbalance
d) All of the above

@ ERSpublications

Can you diagnose this rare complication following an endoscopic intervention in this patient with alcohol-related liver cirrhosis presenting with haematemesis and melena? http://ow.ly/z7Yg30jEHxw 


\section{Answer 2}

d) All of the above

Upon identification of cardiac arrest, immediate cardiopulmonary resuscitation (CPR) was given. The patient regained spontaneous circulation with $1 \mathrm{~min}$ of CPR and one injection of adrenaline. Bands were successfully applied on the gastrooesophageal junction. Bedside echocardiography showed elevated right heart pressure consistent with the diagnosis of acute pulmonary embolism. Chest radiography showed perfusion defect in right lung (figure 1). Computed tomography (CT) pulmonary angiography showed filling defects in the main pulmonary arteries and their segmental branches (figures 2-4).

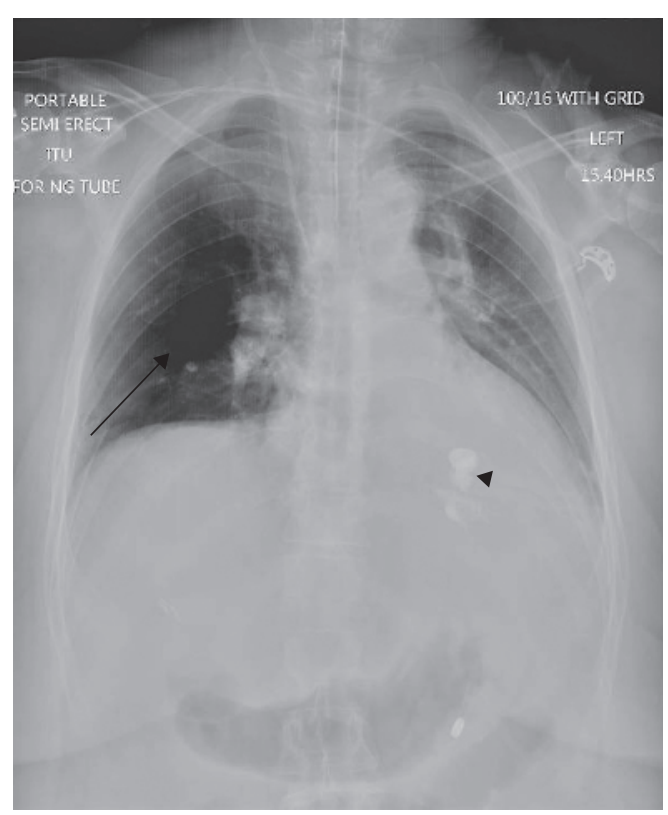

Figure 1 Chest radiograph showing a large perfusion defect in the right lung (arrow) and glue material visible in the stomach (arrowhead).

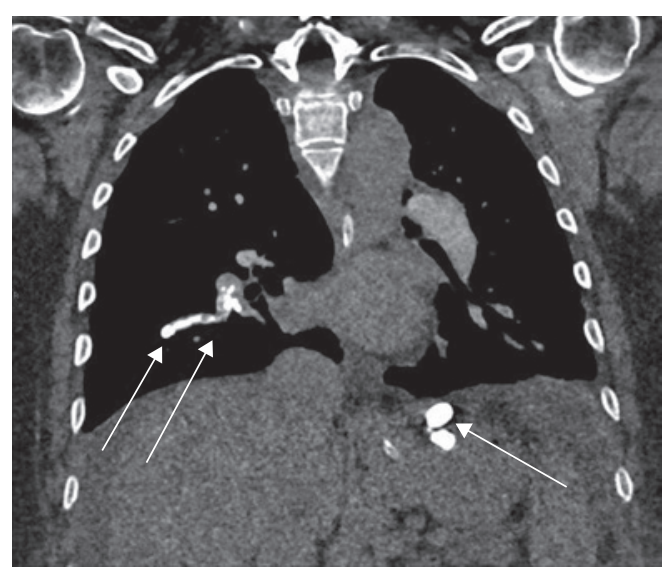

Figure 2 CT pulmonary angiogram showing pulmonary vascular filling defects (arrows).

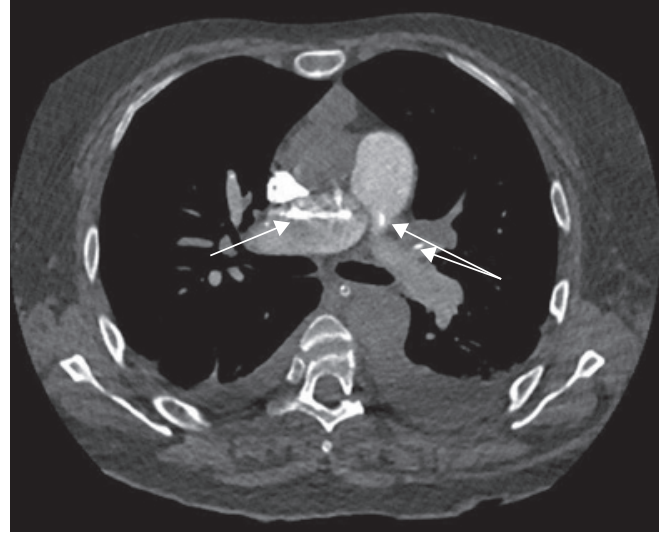

Figure 3 CT pulmonary angiogram showing pulmonary vascular filling defects (arrows).

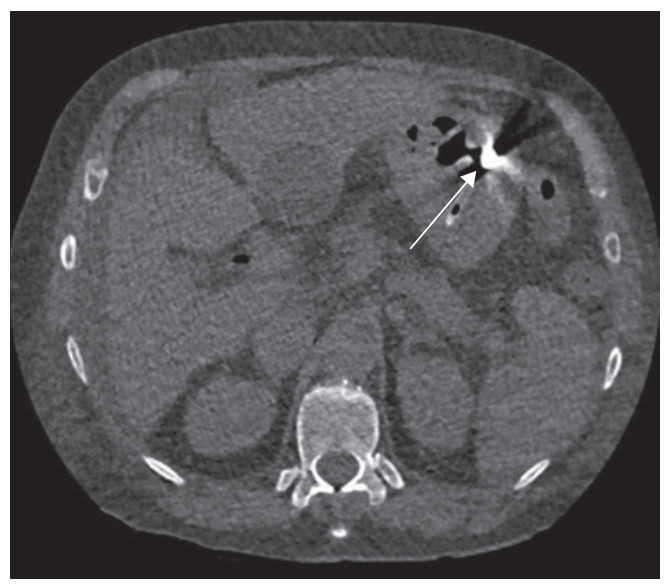

Figure 4 CT pulmonary angiogram showing glue material visible in the stomach (arrow).

\section{Task 3}

What is the most likely diagnosis?
a) Acute pulmonary thromboembolism
b) Acute pulmonary glue embolism
c) Chronic pulmonary thromboembolism
d) Septic emboli
e) None of the above 


\section{Answer 3}

b) Acute pulmonary glue embolism

The clinical features of sudden cardiac arrest, elevated right heart pressures on echocardiography and perfusion defect on chest radiography were all in keeping with an acute pulmonary embolic event. The radiological density of filling defects was consistent with the diagnosis of glue pulmonary embolism. The patient was looked after in the intensive care unit with mechanical ventilation support for 3 days and subsequently transferred to the medical ward for further care. The patient was discharged with no further cardiorespiratory sequelae. 3 months later, a follow-up CT pulmonary angiogram showed resolution of most of the emboli with some residual glue in the right pulmonary vasculature (figure 5).

The tissue glue was a mixture of $N$-butyl-2cyanoacrylate (histoacryl) and the oily contrast agent lipidoil, used in a concentration ratio of $1 / 3$ (histoacryl/lipidoil). The glue polymerises upon contact with the bleeding varix and acts as a tissue adhesive [1]. It has been successfully used in many countries for over two decades as the optimal initial treatment of gastro-oesophageal varices. The use of glue poses a risk of embolic adverse events during or shortly after the procedure. The events reported so far include pulmonary embolism, portal and splenic vein thrombosis, splenic infarction, and recurrent sepsis due to glue septic emboli [2].

The pathophysiology of glue embolism is complex. Injected glue presumably migrates from the gastric varices to the right heart via gastrorenal veins, splenorenal veins and inferior vena cava, and finally lodges into the pulmonary circulation. In case of oesophageal varices, the presumed pathway is

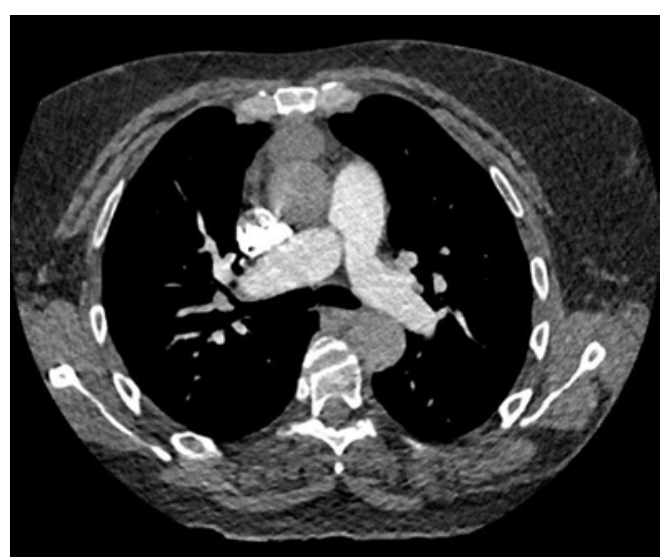

Figure 5 CT pulmonary angiogram (3 months after the embolic event) showing resolved emboli with some residual glue.

through the azygos vein and superior vena cava [3]. The risk factors for glue embolism include the volume of agent, size of varices and speed of injection [4].

The glue embolism is treated by supportive measures and there is no role for anticoagulation or thrombolysis. The emboli spontaneously resolve with time; however, radiological features may persist [4].

Glue embolism is a rare and potentially fatal complication of endoscopic procedure. The presentation of cardiorespiratory compromise can be subacute or delayed, manifesting as pulmonary hypertension, right heart failure or congestive heart failure. It is possible that this condition is underdiagnosed in mildly symptomatic or asymptomatic groups of patients. Further data collection and research are required to establish a preendoscopy risk assessment for potential glue embolic events in the setting of endoscopic procedures.

\section{Acknowledgements}

We would like to thank consultant radiologist Danial Fox (Musgrove Park Hospital, Taunton and Somerset NHS Trust, Taunton, UK) for selecting the best representative CT scan sections for the article.

\section{Conflict of interest}

None declared.

\section{References}

1. Bhat YM, Banerjee S, Barth BA, et al. Tissue adhesives: cyanoacrylate glue and fibrin sealant. Gastrointest Endosc 2013; 78: 209-215

2. Hamad N, Stephens J, Maskell GF, et al. Case report: Thromboembolic and septic complications of migrated cyanoacrylate injected for bleeding gastric varices. BrJ Radiol 2008; 81: e263-e265.
3. Rickman OB, Utz JP, Aughenbaugh GL, et al. Pulmonary embolization of 2-octyl cyanoacrylate after endoscopic injection therapy for gastric variceal bleeding. Mayo Clin Proc 2004; 79: 1455-1458.

4. Alexander S, Korman MG, Sievert W. Cyanoacrylate in the treatment of gastric varices complicated by multiple pulmonary emboli. Intern Med J 2006; 36: 462 